\title{
Concomitant effects of Mindfulness: The Mindfulness Strategies and Difficulties Questionnaire
}

\section{Running title: MSDQ}

\section{Authors}

Ciarán O’Driscoll ${ }^{1,2}$, Aditi Bhansali ${ }^{1,}$, Vaughan Bell ${ }^{1}$ and Madiha Shaikh ${ }^{3,4}$

1 Division of Psychiatry, University College London

2 Institute of Cognitive Neuroscience, University College London

3 Research Department of Clinical, Educational and Health Psychology, University College London

4 Research \& Development Department, North East London NHS Foundation Trust, UK.

*Corresponding author

Dr Ciarán O’Driscoll, UCL Institute of Cognitive Neuroscience, Alexandra House, 17-19 Queen Square,

Bloomsbury, London WC1N 3AZ. Tel: 00447986593045 Email: ucjtcio@ucl.ac.uk

Orchid ID:

Ciaran O’Driscoll https://orcid.org/0000-0002-7316-3041

Vaughan Bell https://orcid.org/0000-0001-8616-4847

Madiha Shaikh https://orcid.org/0000-0003-3362-5691 


\begin{abstract}
Objective: Mindfulness involves a range of cognitive and affective processes. Most research has investigated the 'intended' and beneficial processes. The cognitive-emotional regulatory strategies (CERS) and difficulties encountered during mindfulness practice were investigated in order to establish a questionnaire to evaluate concomitant processes that individuals may engage in during mindfulness. Method: Participants $(n=402)$ completed a survey to measure the frequency of CERS use and difficulties experienced during mindfulness. Items and factor structure were evaluated using multidimensional item response theory. Reliability and validity indices were also investigated. Differences in mindfulness practice across socio-demographic groups, levels of experience were also reported. Results: A 3 factor, 22 item mindfulness strategies and difficulties questionnaire (MSDQ) appears to be reliable and valid in assessing CERS and difficulties experienced during mindfulness practice. The factors were predictive of wellbeing and difficulties of mindfulness success. Level of experience and frequency of practice influenced strategy use and difficulties experienced. Discussion: The study validated the measurement of associated CERS and difficulties in a diverse sample of individuals who practice mindfulness. Results highlighted associated processes which individuals engage in during mindfulness while also emphasizing the context dependence of CERS use.
\end{abstract}

Keywords: mindfulness, emotion regulation; strategies, difficulties 


\section{Introduction}

Mindfulness meditation has emerged from Buddhist meditation that was traditionally practiced as a religious path to “awakening" (Lindahl et al., 2017). It involves the practice of attending, with awareness, to a present moment, while accepting all emotional and cognitive processes, positive or negative, in a non-judgmental fashion (Kabat-Zinn, 1994).

The development and implementation of mindfulness-based interventions (MBIs) in mental health have resulted in a large number of studies recommending the intervention for managing and treating psychological distress and improving quality of life among the wider population. These include; healthy populations (Chiesa \& Serretti, 2009), individuals with mental disorders; namely depression and anxiety (Vøllestad, Nielsen \& Nielsen, 2011; Hofmann, Sawyer, Witt \& Oh, 2010; Teasdale et al., 2000; Strauss, Cavanagh, Oliver \& Pettman, 2014), and individuals with chronic somatic illnesses (Abbott et al., 2014) with mindfulness based cognitive therapy recommended for treatment resistant depression (National Institute for Health and Clinical Excellence, 2016). Moreover, mindfulness is a central component of many "third wave" therapies such as Acceptance and Commitment Therapy, Compassion Focused therapy and Dialectical Behavioural Therapy and has become widespread as a stand-alone aid easily accessed through mobile phone applications and self- help books.

The large proportion of research has focused on evaluating the efficacy of mindfulness-based interventions (Brown \& Cordon, 2009). However, an equally important direction for research is to investigate the underlying processes, which can help elucidate how and why mindfulness works for some and not others. Researchers have stressed the need to describe and operationalize mindfulness and how it works to facilitate our understanding of its functional role in subjective experiences and wellbeing (Brown \& Cordon, 2009). Several theories for the mechanisms underlying mindfulness exist, for instance Shapiro and colleagues (2006) describe successful mindfulness involving intentionally paying attention to experiences in a non-judgmental and accepting manner where intention, attention and attitude are suggested to be the three fundamental dimensions from which other processes emerge during mindfulness. Variations in the dimensions, between individuals, can contribute to the different experiences during mindfulness, and these dimensions can be used to predict how mindfulness works for individuals (Shapiro, Carlson, Astin \& Freedman, 2006). 
Baer and colleagues (2006) developed a model for components of mindfulness, by amalgamating five independently developed measures to draw out the central dimensions, including: 1) observing; 2) describing; 3) acting with awareness; 4) non-judging of inner experiences; and 5) non-reactivity to inner experiences. The more 'facets' used by an individual, the more experienced or successfully they are considered to be engaging in mindfulness (Baer et al., 2006). Baer et al., (2006) propose that variations in the use of these facets could explain differences in the outcomes of mindfulness. Moreover, Holzel and colleagues (2011) identified components of mindfulness from different populations, which included; attention regulation, body awareness, emotion regulation, reappraisal and change in perspective on the self which they suggest work synergistically.

There is currently a paucity of work on the negative predictors of successful mindfulness practice and outcome. Qualitative research into the experience of meditators suggest that there may be a number of adverse emotional responses such as anxiety or loss of affect with meditators reporting experience can be exposing or leave them feeling disorientated (Lindahl, 2017). Crane and Williams (2010) noted some individuals report feeling distracted by negative emotions and depressive thoughts during mindfulness and this may worsen symptoms of depression. The processes of mindfulness require sustained attention to the present moment and cognitive effort to maintain present moment awareness may come at a cost where individuals may experience difficulties, for instance reducing self-regulatory strength (Evans et al. 2014) or increasing physiological stress response (Creswell et al. 2014). The practice of nonjudgemental awareness has also been implicated in affecting cognitive processing, where it has been shown that practicing mindfulness may affect reality-monitoring accuracy, increasing false-memory susceptibility (Wilson et al., 2015).

One way of conceptualizing these processes is through the lens of cognitive-emotional regulatory strategies (CERS; Gross, 1998). CERS are a set of processes responsible for maintaining optimal homeostatic arousal, engaged in relation to primarily undesirable or unwanted internal experiences (Gross, 1998). These strategies have adaptive and maladaptive qualities which need to be evaluated in relation to the context (Aldao, 2013, Brockman et al, 2016). These strategies may not be helpful when engaging in mindfulness or for wellbeing for instance; distraction, relaxation (Baer, 2003), mind-wandering (Mrazek, Smallwood, \& Schooler, 2012), dissociation (Baer, 2006) active suppression, rumination or worrying (Guendelman, Medeiros, \& Rampes, 2017). CERS have been implicated in mental health disorders (Aldao et al., 2010; O’Driscoll et al., 2014) and individuals experience challenging and difficult periods during mindfulness as they use CERS as these may increase psychological distress (Hayes, 2004). 
Nevertheless, psychometric measures of the components of mindfulness practice and experience currently fail to capture the unintended strategies and experiences encountered during mindfulness. To this end, the current study reports the design and initial validation of the "Mindfulness Strategies and Difficulties Questionnaire" (MSDQ) - a multidimensional measure that attempts to capture both useful strategies and difficulties encountered during mindfulness.

This study took a two stage approach. Firstly, the assessment of the validity and reliability of the MSDQ questionnaire was addressed through multidimensional item response theory (MIRT). We also expected to find theoretically meaningful associations between the factors of the MSDQ, and the validated Five Facet Mindfulness Questionnaire (FFMQ) in an effort to elucidate the experience of mindfulness.

Second, this study explored the relationship between the MSDQ, demographic features (e.g., age, religion, ethnicity), practice variables (experience level, form of practice, intensity) and wellbeing, as these features may influence the experience of mindfulness.

This study contributes to existing research on mindfulness by shedding light on the relevance of the unintended strategies that an individual may engage in, and the difficulties encountered during mindfulness.

Moreover, the MSDQ questionnaire will hopefully serve as an adjunctive measure to capture these concomitant processes, and thereby developing a broader understanding of the construct of mindfulness. A more developed understanding of the underlying mechanisms underpinning mindfulness will facilitate applied research and the psychotherapeutic use of mindfulness.

\section{Method}

\section{Procedure}

Adult participants (over 18 years old) with experience of practicing mindfulness, self-selected to participate via social media platforms (i.e. Facebook, Twitter) and snowball sampling. A student sample was approached through 
university participant pathways. In addition, organizations associated with meditation and mindfulness of all levels (beginners-advanced), were contacted to distribute the survey to their members. The study was approved by the UCL Research Ethics Committee (Project ID: 10347/001). Participants completed the survey anonymously using Opinio.

\section{Participants}

A total of 402 participants completed the questionnaires. Participants included 113 males and $289(72 \%)$ females aged 18 to 72 years $($ Mean $=33.82, \mathrm{SD}=12.07)$. A majority of participants were Asian/Asian British $(\mathrm{n}=236)$ ethnicity and from Europe $(n=230)$. The most common religion followed was Jainism $(n=133)$. A more detailed summary of demographics are presented in the appendix. Practice related variables were also collected. These included 1) experience: how long individual has been practicing mindfulness for 2) frequency of formal mindfulness practice 3) frequency of informal mindfulness practice and 4) intensity: length of practice sessions 5) source: how the individual 'learned' mindfulness.

\section{Measures}

The 'Mindfulness strategies and difficulties Questionnaire (MSDQ) (see appendix) was developed to identify difficulties and subjective utility (strategies) of mindfulness practice. Items were generated based on screening of the relevant literature and selected and revised through peer review by a panel familiar with measurement of emotion regulation and delivering mindfulness based interventions. Items were developed to correspond with the process model of emotional regulation (Gross, 1998, Gratz \& Roemer, 2004; Webb et al., 2012) including dissociative responses (Zerubavel \& Messman-Moore, 2015) and difficulties related to mindfulness related obstacles (Graves et al., 2015). Each item was measured on a four point Likert. The items aim to identify distraction, rumination, worry, suppression, relaxation, mind wandering and also dissociative responses. Difficulties relate to affective and attentional processes. Identified strategies are not in themselves inherently maladaptive, however, they are not the explicated intention of mindfulness. Intended processes were not investigated within this measure as these form the basis of existing mindfulness process measures. This resulted in a pool of 26 items.

The Five Factor Mindfulness Questionnaire (Baer et al., 2006): A well-validated standardized questionnaire yielding five factors from five independent studies that represent how mindfulness is currently conceptualized. The five facets include; observing, describing, acting with awareness, non-judging of inner experience, and non-reactivity to inner 
experience. Internal consistency for the five facets ranged from.72 to .92 . A total score of 195 where higher scores reflect high levels of mindfulness.

The Short Warwick-Edinburgh Mental Wellbeing scale (SWEMWBS) (Tennant et al., 2007): A well-validated standardized questionnaire to measure mental wellbeing in the general population. It is a 7-item scale where the items are all worded positively and cover both feeling and functioning aspects of mental wellbeing. Internal consistency was .845 . Total score of 35 where higher score indicates greater overall mental well-being.

\section{Data Analysis}

Analysis of the item pool was conducted using multidimensional item response theory (MIRT). MIRT undertakes a process not dissimilar to factor analysis, while also including item and model fit estimation (Chalmers, 2012). The number of factors was investigated using the non-graphical solutions to scree test (Raiche \& Magis, 2015). Exploratory MIRT using oblimin rotations was used to investigate the factor structure of MSDQ, item selection and discrimination.

A confirmatory model was subsequently used to assess fit and reliability. Reliability was assessed using a MIRTbased index: empirical reliability (a calculation of latent trait estimates and their associated standard errors using a Bayesian modal estimation procedure). To establish convergent validity, the factor loadings, and the average variance extracted (AVE) for each subscale were considered (Henseler et al., 2015).

Construct and divergent validity were investigated through the Pearson correlations between the MSDQ subscales, SWEMWBS and FFMQ. Concurrent validity was explored through the subscales' ability to distinguish between novice and more experienced meditators.

To complement the reliability and validity analysis, and establish predictive validity, a graphical 'least absolute shrinkage and selection operator' (glasso) network model (Epskamp et al., 2012) was conducted. A partial correlation network is similar to the predictive associations obtained in multiple regression and thus, the strength of a connecting line (edge) indicates the strength of the predictive relationship between two dimensions. Predictive validity was supported by regression modelling. Finally, associations between subscales and the demographic and practice related variables were explored. Analyses were conducted in SPSS and R (R Core Team, 2013). 


\section{Results}

\section{Evaluation of measurement model}

The tests for assumptions of items were run and considered adequate; Kaiser-Meryer-Olkin (0.872), Bartlett's test of sphericity $(\chi 2(325)=3994.176, p<0.001)$ showed good fit to the data. Initial factor investigation suggested a 2 or 3 factor solution. Items 2 ('It helps me to doze off or fall asleep') and 5 ('It allows my mind to wander and daydream') of CERS and item 12 ('I blank out') of difficulties had communality value of less than 0.3 and were removed. Item 9 ('It allows me to mind to be more creative or generate ideas') was removed due to cross loading. The remaining items fit into 3 factors (see Table 1). The first factor composed of the difficulties items capturing reactions such as boredom, fatigue and disappointment, the second factor was labeled as 'engaging' CERS as items capture processes relating to rumination and worry and the third factor as 'distancing' CERS capturing processes of suppression and distraction.

\section{Reliability}

All indicators indicated acceptable model fit to this 3 factor structure: $\chi 2(131)=425.4,(p<0.001), S R M S R=0.13$, $\mathrm{CFI}=0.95, \mathrm{TLI}=0.95$, and RMSEA $=0.064(\mathrm{CI} 95 \%=0.056-0.071)$. Internal consistency was good with high subscale empirical reliability values (difficulties: 0.87, engaging: 0.84, distancing: 0.84 ). Item discrimination ranged from moderate to very high; suggesting that individual items are reliable. 


\begin{tabular}{|c|c|c|c|c|c|c|c|c|}
\hline & F1 & F2 & F3 & h2 & MDISC & b1 & $\mathrm{b} 2$ & b3 \\
\hline CER1 & & & 0.608 & 0.370 & & & & \\
\hline CER3 & & & 0.590 & 0.348 & 1.30 & -1.67 & 0.20 & 1.57 \\
\hline & & & & & 1.24 & -2.91 & -0.87 & 0.79 \\
\hline CER4 & & & 0.712 & 0.507 & 172 & 116 & 005 & 156 \\
\hline CER6 & & & 0.735 & 0.540 & 1.13 & -1.16 & 0.25 & 1.56 \\
\hline & & & & & 1.84 & -1.27 & 0.13 & 1.43 \\
\hline CER7 & & 0.690 & & 0.476 & 1.62 & -4.55 & -0.73 & 0.52 \\
\hline CER8 & & & 0.754 & 0.568 & 1.95 & -1.16 & 0.19 & 1.69 \\
\hline CER10 & & 0.723 & & 0.522 & 1.78 & -1.07 & 0.17 & 1.29 \\
\hline CER11 & & 0.873 & & 0.763 & 3.05 & -3.45 & -0.83 & 0.19 \\
\hline CER12 & & 0.888 & & 0.788 & 3.28 & -3.39 & -1.05 & -0.01 \\
\hline CER13 & & & 0.787 & 0.620 & 2.17 & -3.82 & -0.90 & 0.50 \\
\hline CER14 & & & 0.764 & 0.583 & 2.01 & -1.21 & 0.33 & 1.57 \\
\hline d1 & 0.755 & & & 0.570 & 1.96 & -0.30 & 1.30 & 2.46 \\
\hline $\mathrm{d} 2$ & 0.601 & & & 0.362 & 1.28 & -1.48 & 0.53 & 2.25 \\
\hline d3 & 0.710 & & & 0.505 & 1.72 & -0.25 & 1.03 & 2.46 \\
\hline d4 & $\begin{array}{l}0.711 \\
0.758\end{array}$ & & & $\begin{array}{l}0.506 \\
0.575\end{array}$ & 1.72 & -0.57 & 0.81 & 1.90 \\
\hline d6 & 0.830 & & & 0.689 & 1.98 & 0.47 & 1.58 & 2.90 \\
\hline d7 & 0.705 & & & 0.497 & 2.54 & 0.28 & 1.35 & 2.49 \\
\hline d8 & 0.707 & & & 0.50 & 1.69 & -0.18 & 1.18 & 2.33 \\
\hline d9 & 0.691 & & & 0.477 & 1.70 & -0.53 & 1.28 & 2.43 \\
\hline & & & & & 1.63 & -1.22 & 0.65 & 2.00 \\
\hline d10 & 0.662 & & & 0.438 & 1.50 & -0.29 & 1.38 & 2.64 \\
\hline d11 & 0.643 & & & 0.414 & 1.43 & -0.59 & 1.03 & 2.25 \\
\hline
\end{tabular}

Table 1 MIRT analysis for the questionnaire 


\section{Validity}

Standardized loadings of items were all higher than .50. Item loadings (oblimin rotation method) ranged between 0.59 and 0.888 . Discrepancies between cross loadings were large (>.4) for all items. Average variance extracted (AVE) for each factor, indicated that at least $50 \%$ (50\% to 64\%) of the variance in each factor was due to the hypothesized underlying trait. These results support the factor based convergent validity of the model.

It was expected that novices and more experienced meditators would differ on difficulties and CERS. According to Welch's t-test, more experienced mediators experienced fewer difficulties $(\mathrm{t}(377.2)=4.996, p<.001, \mathrm{~d}=0.497)$ and more use of distancing CERS ( $\mathrm{t}(398.9)=-2.057, p=.04, \mathrm{~d}=0.205)$. No significant difference was noted for engaging CERS.

Correlations (see Table 2) were in line with theoretical expectations supporting construct and divergent validity. Difficulties was also negatively correlated with all FFMQ dimensions. Both CERS were positively correlated with observing and non-reactivity to inner experience. Engaging was positively correlated with awareness and nonjudgement.

\begin{tabular}{|c|c|c|c|c|c|c|c|c|c|}
\hline & $\begin{array}{l}\text { Mean } \\
\text { (SD) }\end{array}$ & diff & Dist & Eng & Well & Obs & Desc & Aware & $\begin{array}{c}\text { non } \\
\text { judge }\end{array}$ \\
\hline Difficulties & $\begin{array}{r}22.09 \\
(6.6)\end{array}$ & - & & & & & & & \\
\hline Distancing & $\begin{array}{r}17.13 \\
(4.5)\end{array}$ & $-0.241^{* * * *}$ & - & & & & & & \\
\hline Engaging & $\begin{array}{r}9.39 \\
(3.17)\end{array}$ & -0.030 & $0.452 * * *$ & - & & & & & \\
\hline Wellbeing & $\begin{array}{l}24.57 \\
(5.04)\end{array}$ & $-0.339 * * *$ & $0.358 * * *$ & $0.251 * * *$ & - & & & & \\
\hline Observing & $\begin{array}{l}24.78 \\
(5.93)\end{array}$ & $-0.193 * * *$ & $0.182 * * *$ & $0.156^{* *}$ & $0.272 * * *$ & - & & & \\
\hline Describing & $\begin{array}{l}24.67 \\
(5.03)\end{array}$ & $-0.159 * *$ & 0.087 & 0.053 & $0.302 * * *$ & $0.413 * * *$ & - & & \\
\hline Awareness & $\begin{array}{l}22.84 \\
(4.52)\end{array}$ & $-0.187 * * *$ & -0.047 & $-0.156 * *$ & $0.125^{*}$ & 0.066 & $0.186^{* * *}$ & - & \\
\hline nonjudgement & $\begin{array}{l}22.14 \\
(4.76)\end{array}$ & $-0.129 *$ & -0.04 & $-0.116^{*}$ & 0.104 & -0.013 & $0.195 * * *$ & $0.458 * * *$ & - \\
\hline Non reacting & $\begin{array}{l}20.07 \\
(4.95)\end{array}$ & $-0.245^{* * *}$ & $0.263 * * *$ & $0.251 * * *$ & $0.478 * * *$ & $0.569 * * *$ & $0.449 * * *$ & 0.082 & $0.108^{*}$ \\
\hline
\end{tabular}

Table 2: Mean and standard deviations for questionnaires and Pearson correlation matrix 
Predictive validity was explored through complementary network and regression analysis. The partial correlation network was generated using Extended Bayesian Information Criterion (EBIC), the hyperparameter was set at 0.5 for a more parsimonious model. Analysis was based on pairwise complete observations. Strength centrality (Opsahl et al., 2010) quantifies how much influence a dimension has on the rest of the network. Correlation stability coefficients computed for strength centrality metrics (0.594), surpassed the recommended cut off of 0.25 (Epskamp et al., 2017) and were therefore interpretable.

The network (Figure 1) displays the influence that each dimension has on other dimensions within the network when controlling for the influence of all other dimensions in the network. Of interest, are the associations between MSDQ dimensions and mindfulness dimensions (when controlling for wellbeing), and the relationship the MSDQ has with wellbeing. The resulting associations specify direct associations between the MSDQ dimensions and wellbeing. Only two of the FFMQ dimensions had direct associations with wellbeing (non reactivity and describing).

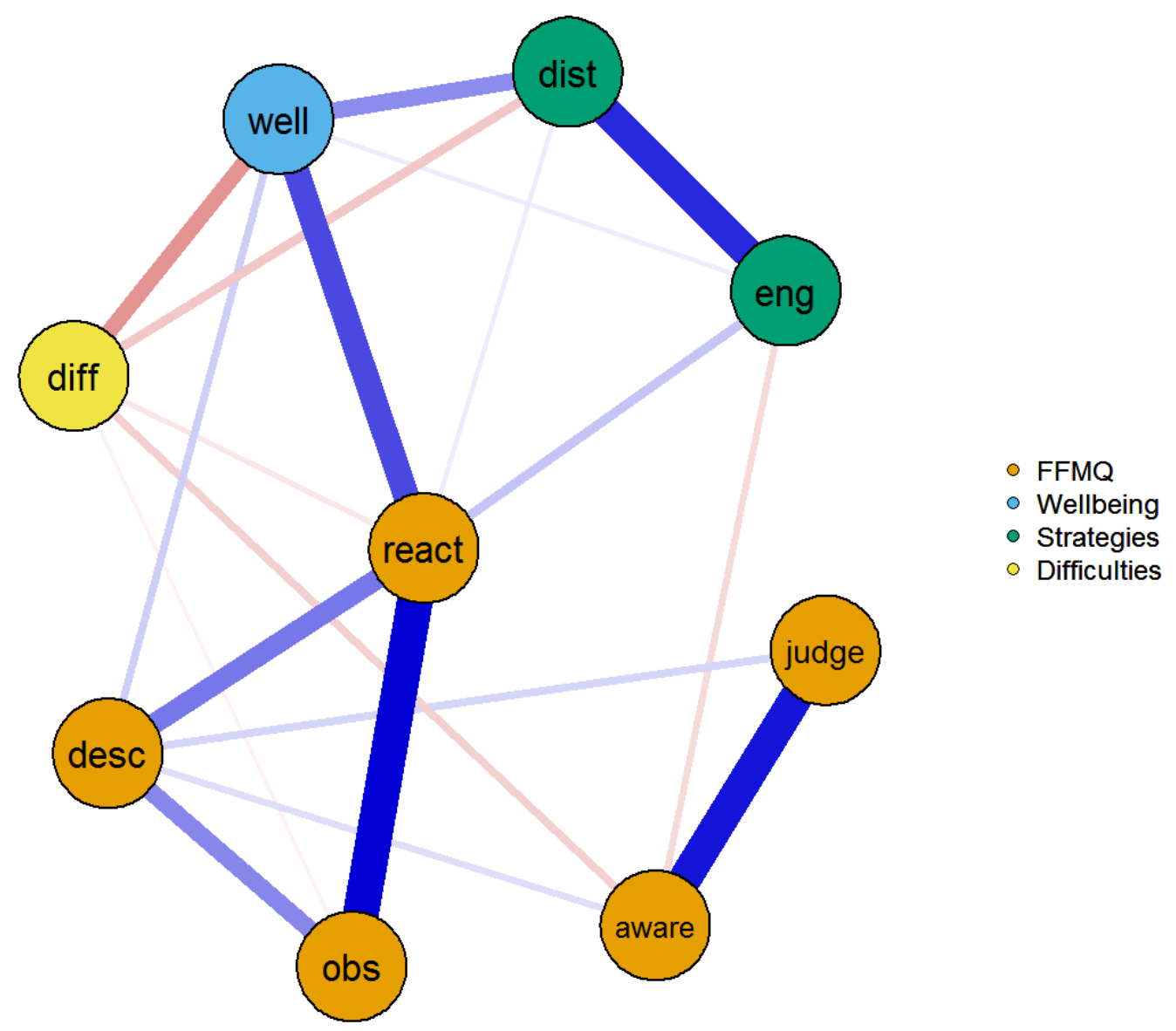

Fig 1 Network of associations (glasso) between the dimension of the FFMQ and the MSDQ questionnaires. 
Non-reactivity displayed highest strength, suggesting a central role in mindfulness. This dimension showed strong positive associations with engaging strategies, and describing. There was a weak negative association between non-reactivity and difficulties. Awareness was negatively associated with engaging and difficulties. This analysis reveals the dynamic association between dimensions, in addition it helps to elucidate the relationship between MSDQ factors, where distancing moderates the relationship between difficulties and engaging.

The network identified the variables to be included in our regression models. We chose to compare two models; the MSDQ variables alone and alongside reactivity and describing, as predictors of wellbeing. The model comprising of the MSDQ factors alone $\mathrm{F}(3,346)=31.69, p<.001, \mathrm{R}^{2}$ adj $=0.209$ suggested that all variables are significant predictors of wellbeing (Table 3 ). The combined, model displayed a large association $\mathrm{F}(5,344)=$ $36.11, p<.001, \mathrm{R}^{2}$ adj $=0.335$ represented a $\mathrm{R}^{2}$ change of 0.129 compared to MSDQ alone.

MSDQ factors were entered into a regression model to determine their relationship to mindfulness success as quantified by the FFMQ total score (McDonald's $\omega=0.878$ ). The model displayed a significant association $\mathrm{F}(3,352)=11.72, p<.001, \mathrm{R}^{2}$ adj $=0.091$, however difficulties was the only significant variable, suggesting it as an independent predictor of mindfulness success.

\begin{tabular}{llrrrrr}
\hline & MSDQ & \multicolumn{1}{l}{ S } & & \multicolumn{1}{c}{$\beta$} & \multicolumn{1}{c}{$t$} & \multicolumn{1}{c}{$p$} \\
\hline \multirow{4}{*}{ Wellbeing } & & & & & & \\
& Distancing & 0.253 & 0.062 & 0.223 & 4.07 & $<.001$ \\
& Difficulties & -0.246 & 0.042 & -0.287 & -5.808 & $<.001$ \\
& Engaging & 0.247 & 0.085 & 0.154 & 2.898 & 0.004 \\
\hline \multirow{2}{*}{ Mindfulness } & & & & & & \\
success & Distancing & 0.221 & 0.212 & 0.061 & 1.042 & 0.298 \\
& Difficulties & -0.729 & 0.142 & -0.271 & -5.127 & $<.001$ \\
& Engaging & 0.23 & 0.289 & 0.045 & 0.795 & 0.427 \\
\hline
\end{tabular}

Table 3: Regression Table. 


\section{Demographics}

For the purposes of establishing any differences in difficulties, engaging and distancing strategies based on demographic variables, independent samples t-test (gender), correlation (age) and one-way ANOVAs (religion, ethnicity) were conducted.

Comprehensive descriptive data can be found in supplementary information, summary data for questionnaires are presented in Table 2. Due to large number of levels in each demographic variable, we reduced the number of levels. Reduction of such variables was based on major groupings existing in each variable, Ethnicity (Asian/Asian British, White, Other ethnicity), religion (Jainism, Judeo-Christian, no religion, Hinduism and other religions).

No significant group differences were noted for gender. Difficulties was the only factor associated with age $(\mathrm{r}=$ 0.153, $\mathrm{p}=0.002$ ), where increasing age was related to experiencing less frequent difficulties. However, age was also significantly correlated with years of experience $(r=0.405, \mathrm{p}<0.001)$.

There was a significant effect of religion on distancing and engaging strategy use $(\mathrm{F}(4,381)=4.04, \mathrm{p}=0.003$, and $\mathrm{F}(4,381)=7.13, \mathrm{p}<0.001$ respectively). Post hoc comparison (Tukey HSD test) indicated that higher level of distancing strategies were engaged by Jainism participants than Judeo-Christian $\left(\mathrm{M}_{\mathrm{diff}}=2.04, \mathrm{SE}=0.69\right)$ and no religion $\left(\mathrm{M}_{\mathrm{diff}}=2.13, \mathrm{SE}=0.6\right)$ participants. A similar difference was noted for engaging strategies, where participants with no religion reported lower use of engaging strategies than Hindu $\left(\mathrm{M}_{\mathrm{diff}}=2.17, \mathrm{SE}=0.50\right)$, Jainism $\left(\mathrm{M}_{\text {diff }}=1.76, \mathrm{SE}=0.42\right)$ and 'other' religions $\left(\mathrm{M}_{\text {diff }}=1.92, \mathrm{SE}=0.61\right)$ participants. Hindu participants also reported higher engaging strategy use than Judeo-Christian participants $\left(\mathrm{M}_{\mathrm{diff}}=1.51, \mathrm{SE}=0.55\right)$. Ethnicity difference tended to reflect the above associations, with a significant effect on distancing $(F(2,399)=5.87, p=0.003)$ and engaging $(\mathrm{F}(2,399)=10.46, \mathrm{p}<0.001)$ strategy use with Asian/Asian British reporting greater CERS use than Caucasian participants (distancing: $\mathrm{M}_{\mathrm{diff}}=1.66, \mathrm{SE}=0.49$; engaging: $\mathrm{M}_{\mathrm{diff}}=1.541, \mathrm{SE}=0.34$ ).

\section{Practice related predictors of MSDQ}

Multiple regression analysis was conducted to examine the relationship between MSDQ factors and potential practice related predictors. Frequency of formal and informal practice were entered into a multiple regression for each of the MSDQ factors. 
The way participants learned mindfulness (self guided, practitioner led or though associated practice) was not associated with factors from the MSDQ or FFMQ. More difficulties was associated with more frequent informal $\operatorname{practice}(\beta=0.12, \mathrm{t}=2.469, \mathrm{p}=0.014) ; \mathrm{F}(2,399)=22.96, \mathrm{p}<.001, \mathrm{R}^{2}{ }_{\text {adj }}{ }^{=} 0.099$. Lesser use of distancing strategies was associated with greater frequency of formal practice $(\beta=-0.186, \mathrm{t}=-3.793, \mathrm{p}<0.001)$. Lesser use of engaging strategies was associated with greater frequency of both informal $(\beta=-0.136, \mathrm{t}=-2.634, \mathrm{p}=0.009)$ and formal $\operatorname{practice}(\beta=-0.126, \mathrm{t}=-2.432, \mathrm{p}=0.015) ; \mathrm{F}(2,399)=9.595, p<.001, \mathrm{R}^{2}{ }_{\text {adj }}=0.041$

\section{Discussion}

This study reports on the preliminary validation of the MSDQ, a measure of CERS and difficulties experienced during mindfulness. MIRT analysis suggested three factors (22 items): engaging and distancing CERS use and difficulties experienced during mindfulness. The MSDQ displayed good model fit, internal consistency and validity, supporting the utility to assess concomitant processes of mindfulness practice and providing evidence that the items represent a reasonable measure of the construct that can be used with some confidence for further data collection. The initial validation highlights the dynamic interactions between processes underlying mindfulness, the MSDQ as a predictor of wellbeing and a clear role for difficulties affecting successful engagement in mindfulness.

The CERS factors identified correspond with previous theories (Hayes, 2004; Gross, 1998), 'engaging' CERS indicative of rumination and worry processes, and 'distancing' indicative of suppression and distraction processes. Positive associations between distancing and engaging strategies and non-reactivity and positive predictive relationship between CERS and wellbeing runs contrary to our understanding of these CERS as experienced in daily life where they would be considered maladaptive and associated with psychopathology. However, the interactions appear to be complex and require validation in other samples. The network model highlights that while engaging strategy use may be positively associated with non-reactivity it is associated with reduced awareness, moderating the association between those two dimensions of mindfulness. While generally considered maladaptive, current thinking suggests CERS use is context dependent (Aldao, 2013). It is interesting therefore to consider how they may be adaptive within the context of mindfulness. It is possible that practice allows for reflection or problem solving, rather than 'brooding' over negative emotion (Treynor et al. 2003). Indeed, research suggests that focusing one's attention (on a concern) while keeping a distance from thoughts or feelings 
influences the adaptiveness of the CERS (Ishikawa et al., 2017). Mindfulness may also facilitate greater executive functioning allowing an individual to think more flexibly (Taren et al., 2017).

Non-reactivity to inner experience appears to be the most important aspect of mindfulness practice, however, the dimensions of the FFMQ and MSDQ, in this sample, did not appear to encapsulate a unified construct of mindfulness. Difficulties during practice may mediate many associations between factors. However, addressing difficulties may not have much impact on the other variables in the network (given its low strength within the network), suggesting that it may not be a suitable target during training. The findings from this network model for the FFMQ show some inconsistency with the original FFMQ validation (Baer et al., 2006) where they highlighted observing as being less associated with the other factors and associated constructs. In this network, non-reactivity and observing are closely linked. Previous factor analytic studies have highlighted that the observing factor differs between meditators and non-meditators (Bauer et al., 2006; Aguado et al., 2015; Williams et al., 2014). As with the CERS, observing, where conceptualized as self-focused attention, may be maladaptive and operationalized differently at different levels of experience (Bauer et al, 2008). Indeed, within this study observing and length of experience were moderately correlated $(r=0.23, \mathrm{p}<0.001)$.

The influence of practice related variables on factors of the MSDQ surprisingly revealed that the way the sample learned mindfulness (self-guided, practitioner led or though associated practice) did not appear to contribute to any factor of the MSDQ, suggesting that other factors are more important. Higher levels of engaging was associated with greater length of experience and lower frequency of practice (both formal and informal) while higher levels of use of distancing strategies was associated with lower frequency of formal practice. Greater difficulties experienced during mindfulness was related to a lower length of experience but more frequent informal practice.

No differences were noted between the way individuals' learned mindfulness on CERS or difficulties. It was assumed that practitioner facilitated learning would allow for sharing of experience to circumnavigate difficulties and specify intended over non-intended processes. It may be that all forms of learning utilize guided audio recordings of formal and/or informal practice which provide a standardized format to the learning experience. Both formal and informal practices appear to reduce engaging CERS use during mindfulness. It is unclear how this may relate to CERS use in day to day life. Research seems to suggest that formal practice confers benefits 
over informal in reducing rumination (Hawley et al., 2013; Chambers, Lo \& Allen, 2007). The association between informal practice and greater difficulties may suggest that the structure supported by formal practice and reduced contact with external stimuli may play a role in reducing difficulties associated with practice.

This pattern of associations between MSDQ factors and practice variables may suggest that engagement in these processes during mindfulness may be advantageous in facilitating successful engagement in mindfulness. This is evidenced by the increasing engagement in processes with level of experience. It is possible that high levels of CERS in experienced and novice meditators reflected different processes (Baur et al., 2006). For instance, with more experience, a meditator may have improved emotional regulation, reducing the experience of negative affect during mindfulness (Jha et al., 2010) and attending to distancing and engaging CERS during mindfulness may not be in reaction to negative affect. It is also possible that different patterns of mindfulness could reveal more nuanced relationships. Profile analysis studies have highlighted that low mindfulness and being 'judgmentally observing' (high on observing, but low on non-judging and acting with awareness) was associated with less adaptive outcomes (Pearson et al., 2015, Bravo, Boothe \& Pearson, 2015).

Religious and ethnic differences were noted on the MSDQ factors where higher reported levels on distancing and engaging CERS use were reported for Eastern (Jainism and Hinduism) over Western (Judeo-Christian) religions and no religion. This is unsurprising given that mindfulness was developed out of Eastern religious practice and is associated with an Eastern cultural view. Contemplative prayer (as in Judeo Christian practice) shares commonalities with meditative practice (Stratton, 2015) however, it is possible that the cultural context may have an additive benefit in development of dispositional mindfulness.

\section{Limitations and Future Directions}

While the study provides preliminary evidence for the construct validity and internal reliability of the MSDQ, further research concerning the psychometric features are indicated. Findings from current measures are restricted by the epistemological stance taken, which may lead participants to discern the social desirable response: doing mindfulness 'right'. While we attempted to circumvent this; by delivering the MSDQ before the FFMQ, meditators are likely to be practiced in the language of mindfulness. 
This study was a cross-sectional analysis thus we were unable to capture test-retest reliability and the relationship with wellbeing is not directed. It would also be of great interest to validate the measure in clinical samples, and across the process/stages of learning mindfulness. This would be helpful where there may be confusion between processes, for instance dissociation and mindfulness both involve the capacity to take the observer position (Zerubavel \& Messman-Moore, 2015) and as such clients may confuse mindfulness meditation with dissociation (Linehan 1993). While we captured CERS during mindfulness we did not assess CERS in daily life. This would have allowed us to understand the participants' mindfulness CERS within the context of their everyday CERS and see how individuals with different emotional regulation styles engage in mindfulness.

The item pool prior to participant completion was already relatively brief compared to other questionnaire designs, and as such, there is the possibility that other unintended processes were overlooked in the development of the MSDQ. However, the brevity of the measure is its strength as shorter measures are more feasible, can save time in clinical settings and increase participation in future research. An additional strength of our study is that it reached a diverse population covering a wide spread of countries from Europe, Asia, and North America. This representative sample improves the generalizability of our findings to the wider population and cross-cultural applicability.

Based on the findings, we suggest that the MSDQ questionnaire is not assessing 'maladaptive' strategies per se, CERS during mindfulness appear to be adaptive within the context of 'successfully' practicing mindfulness. The MSDQ captures an insight into unintended, strategies use and difficulties experienced during mindfulness, that seem to be helping individuals to successfully engage in mindfulness and potentially improving mental wellbeing.

\section{Implications}

The current study helps to operationalize a number of associated processes that individuals may engage in during mindfulness practice which have not been captured in available measures of mindfulness. While in daily life, high engagement with these processes may be related to psychopathology, in the context of mindfulness they may serve an adaptive function allowing a degree of flexibility. This highlights mindfulness practice allowing the context to engage in flexibily responding to internal stimuli, however, this may not reflect engagement in 'mindfulness' as prescribed by practitioners. 
The difficulties subscale highlights that meditators experience challenges during mindfulness practice and the network analysis suggests that these difficulties are associated with other components of mindfulness practice. Though the findings in this study are modest in relation to the influence of difficulties on other components, it is likely that these associations would strengthen in beginner or novice meditators. As such, it is likely to be useful to capture and address these difficulties as they arise in teaching mindfulness.

The MSDQ assesses the frequency of the unintended regulatory strategies and difficulties experienced during mindfulness - aspects of mindfulness that have not been captured by current psychometric tools. Given the relevance of these strategies and difficulties to mindfulness and mental wellbeing, we stress the importance of capturing them, alongside the intended strategies measured in current psychometric tools for mindfulness. By considering a wide range of strategies and experiences encountered in mindfulness, we can gain a more thorough assessment of how individuals practice mindfulness and thus refine our understanding of the adaptive and maladaptive components and how mindfulness works.

\section{References}

Abbott, R., Whear, R., Rodgers, L., Bethel, A., Thompson Coon, J., \& Kuyken, W. et al. (2014). Effectiveness of mindfulness-based stress reduction and mindfulness based cognitive therapy in vascular disease: A systematic review and meta-analysis of randomised controlled trials. Journal Of Psychosomatic Research, 76(5), 341-351. http://dx.doi.org/10.1016/j.jpsychores.2014.02.012

Aguado, J., Luciano, J. V., Cebolla, A., Serrano-Blanco, A., Soler, J., \& García-Campayo, J. (2015). Bifactor analysis and construct validity of the five facet mindfulness questionnaire (FFMQ) in non-clinical Spanish samples. Frontiers in Psychology, 6, 404. http://doi.org/10.3389/fpsyg.2015.00404

Aldao, A. (2013). The future of emotion regulation research: Capturing context. Perspectives on Psychological Science, $8(2), 155-172$.

Aldao, A., Nolen-Hoeksema, S., \& Schweizer, S. (2010). Emotion-regulation strategies across psychopathology: A meta-analytic review. Clinical psychology review, 30(2), 217-237. 
Baer, R. A. (2003). Mindfulness training as a clinical intervention: A conceptual and empirical review. Clinical psychology: Science and practice, 10(2), 125-143.

Baer, R., Smith, G., Hopkins, J., Krietemeyer, J., \& Toney, L. (2006). Using Self-Report Assessment Methods to Explore Facets of Mindfulness. Assessment, 13(1), 27-45. http://dx.doi.org/10.1177/1073191105283504

Baer, R. A., Smith, G. T., Lykins, E., Button, D., Krietemeyer, J., Sauer, S., ... \& Williams, J. M. G. (2008).

Construct validity of the five facet mindfulness questionnaire in meditating and nonmeditating samples. Assessment, 15(3), 329-342.

Bravo, A., Boothe, L., \& Pearson, M. (2015). Getting Personal with Mindfulness: a Latent Profile Analysis of Mindfulness and Psychological Outcomes. Mindfulness, 7(2), 420-432. http://dx.doi.org/10.1007/s12671-015$0459-7$

Brockman, R., Ciarrochi, J., Parker, P., \& Kashdan, T. (2016). Emotion regulation strategies in daily life: mindfulness, cognitive reappraisal and emotion suppression. Cognitive Behaviour Therapy, 46(2), 91-113. http://dx.doi.org/10.1080/16506073.2016.1218926

Brown, K. W., \& Cordon, S. (2009). Toward a phenomenology of mindfulness: Subjective experience and emotional correlates. In Clinical handbook of mindfulness (pp. 59-81). Springer New York

Carlson, L. \& Garland, S. (2005). Impact of mindfulness-based stress reduction (MBSR) on sleep, mood, stress and fatigue symptoms in cancer outpatients. International Journal Of Behavioral Medicine, 12(4), 278-285.

Cattell, R. (1966). The Scree Test For The Number Of Factors. Multivariate Behavioral Research, 1(2), 245-276. http://dx.doi.org/10.1207/s15327906mbr0102_10

Chalmers, R. P. (2012). mirt: A multidimensional item response theory package for the R environment. Journal of Statistical Software, 48(6), 1-29. 
Chambers, R., Lo, B., \& Allen, N. (2007). The Impact of Intensive Mindfulness Training on Attentional Control, Cognitive Style, and Affect. Cognitive Therapy And Research, 32(3), 303-322. http://dx.doi.org/10.1007/s10608007-9119-0

Chiesa, A., \& Serretti, A. (2009). Mindfulness-Based Stress Reduction for Stress Management in Healthy People: A Review and Meta-Analysis. The Journal Of Alternative And Complementary Medicine, 15(5), 593-600. http://dx.doi.org/10.1089/acm.2008.0495

Cigolla, F., \& Brown, D. (2011). A way of being: bringing mindfulness into individual therapy. Psychotherapy Research, 21(6), 709-721.

Crane C, Williams MG. (2010) Factors Associated with Attrition from Mindfulness-Based Cognitive Therapy in Patients with a History of Suicidal Depression. Mindfulness. 1: 10-20

Creswell J.D, Lindsay E.K. (2014). How does mindfulness training affect health? A mindfulness stress buffering account. Curr. Dir. Psychol. Sci. 23(6):401-7

de Bruin, E., Topper, M., Muskens, J., Bögels, S., \& Kamphuis, J. (2012). Psychometric Properties of the Five Facets Mindfulness Questionnaire (FFMQ) in a Meditating and a Non-meditating Sample. Assessment, 19(2), 187-197. http://dx.doi.org/10.1177/1073191112446654

Edenfield, T., \& Saeed, S. (2012). An update on mindfulness meditation as a self-help treatment for anxiety and depression. Psychology Research And Behavior Management, 131. http://dx.doi.org/10.2147/prbm.s34937

Epskamp, S., Cramer, A. O., Waldorp, L. J., Schmittmann, V. D., \& Borsboom, D. (2012). qgraph: Network visualizations of relationships in psychometric data. Journal of Statistical Software, 48(4), 1-18.

Epskamp, S., Borsboom, D., \& Fried, E. (2017). Estimating psychological networks and their accuracy: A tutorial paper. Behavior Research Methods. http://dx.doi.org/10.3758/s13428-017-0862-1 
Evans DR, Eisenlohr-Moul TA, Button DF, Baer RA, Segerstrom SC. 2014. Self regulatory deficits associated with unpracticed mindfulness strategies for coping with acute pain. J. Appl. Soc. Psychol. 44(1):23-30

Germer, C. K. (2005). Teaching mindfulness in therapy. Mindfulness and psychotherapy, 1(2), 113-129.

Gratz, K. L., \& Roemer, L. (2004). Multidimensional assessment of emotion regulation and dysregulation: Development, factor structure, and initial validation of the difficulties in emotion regulation scale. Journal of psychopathology and behavioral assessment, 26(1), 41-54.

Graves, J. Immergut, M. \& (Culadasa) Yates, J. (2015) The Mind Illuminated: A Complete Meditation Guide Integrating Buddhist Wisdom and Brain Science Dharma Treasure Press

Gross, J. (1998) The emerging field of emotion regulation: An integrative review Review of General Psychology, 2 (5) pp. 271-299

Guendelman, S., Medeiros, S., \& Rampes, H. (2017). Mindfulness and Emotion Regulation: Insights from Neurobiological, Psychological, and Clinical Studies. Frontiers $\quad$ In $\quad$ Psychology, 8. http://dx.doi.org/10.3389/fpsyg.2017.00220

Hawley, L., Schwartz, D., Bieling, P., Irving, J., Corcoran, K., \& Farb, N. et al. (2013). Mindfulness Practice, Rumination and Clinical Outcome in Mindfulness-Based Treatment. Cognitive Therapy And Research, 38(1), 19. http://dx.doi.org/10.1007/s10608-013-9586-4

Hayes, A. (2004). Clarifying the Construct of Mindfulness in the Context of Emotion Regulation and the Process of Change in Therapy. Clinical Psychology: Science And Practice, 11(3), 255-262. http://dx.doi.org/10.1093/clipsy/bph080

Henseler, J., Ringle, C. M., \& Sarstedt, M. (2015). A new criterion for assessing discriminant validity in variancebased structural equation modeling. Journal of the academy of marketing science, 43(1), 115-135. 
Hofmann, S., Sawyer, A., Witt, A., \& Oh, D. (2010). The effect of mindfulness-based therapy on anxiety and depression: A meta-analytic review. Journal of Consulting And Clinical Psychology, 78(2), 169-183. http://dx.doi.org/10.1037/a0018555

Hölzel, B. K., Lazar, S. W., Gard, T., Schuman-Olivier, Z., Vago, D. R., \& Ott, U. (2011). How does mindfulness meditation work? Proposing mechanisms of action from a conceptual and neural perspective. Perspectives on Psychological Science, 6(6), 537-559.

Ishikawa, H., Mieda, T., Oshio, A. et al. (2017) Mindfulness, 1-8. https://doi.org/10.1007/s12671-017-0797-8

Jha, A., Stanley, E., Kiyonaga, A., Wong, L., \& Gelfand, L. (2010). Examining the protective effects of mindfulness training on working memory capacity and affective experience. Emotion, 10(1), 54-64. http://dx.doi.org/10.1037/a0018438

Kabat-Zinn, J. (1994). Where ever you go, there you are: Mindfulness meditation in everyday life. New York, NY: Hyperion.

Lau, M., Bishop, S., Segal, Z., Buis, T., Anderson, N., Carlson, L., et al. (2006). The Toronto Mindfulness Scale: Development and validation. Journal of Clinical Psychology, 62, 1445-1467.

Lindahl, J., Fisher, N., Cooper, D., Rosen, R., \& Britton, W. (2017). The varieties of contemplative experience: A mixed-methods study of meditation-related challenges in Western Buddhists. PLOS ONE, 12(5), e0176239. http://dx.doi.org/10.1371/journal.pone.0176239

Linehan, M. M. (1993). Skills training manual for treating borderline personality disorder. New York: Guilford.

Mrazek, M., Smallwood, J., \& Schooler, J. (2012). Mindfulness and mind-wandering: Finding convergence through opposing constructs. Emotion, 12(3), 442-448. http://dx.doi.org/10.1037/a0026678 
National Institute for Health and Clinical Excellence (2016) National Collaborating Centre for Mental Health. Depression: Management of Depression in Primary and Secondary Care. Clinical Guideline CG90.

O'Driscoll, C., Laing, J., \& Mason, O. (2014). Cognitive emotion regulation strategies, alexithymia and dissociation in schizophrenia, a review and meta-analysis. Clinical psychology review, 34(6), 482-495.

Opsahl, T., Agneessens, F., \& Skvoretz, J. (2010). Node centrality in weighted networks: Generalizing degree and shortest paths. Social networks, 32(3), 245-251.

Pearson, M. R., Lawless, A. K., Brown, D. B., \& Bravo, A. J. (2015). Mindfulness and emotional outcomes: identifying subgroups of college students using latent profile analysis. Personality and Individual Differences, 76, 33-38. doi:10.1016/j.paid.2014.11.009.

R Core Team, (2013). R: a language and environment for statistical computing. Version 3.1. 3. Vienna, Austria: R Foundation for Statistical Computing; 2015.

Raiche, G., and D. Magis. 2015. R Package ‘nFactors'. Vienna: R Foundation for Statistical Computing.

Raes, F., \& Williams, J. (2010). The Relationship between Mindfulness and Uncontrollability of Ruminative Thinking. Mindfulness, 1(4), 199-203. http://dx.doi.org/10.1007/s12671-010-0021-6

Ramel, W., Goldin, P., Carmona, P., \& McQuaid, J. (2004). The Effects of Mindfulness Meditation on Cognitive Processes and Affect in Patients with Past Depression. Cognitive Therapy And Research, 28(4), 433-455. http://dx.doi.org/10.1023/b:cotr.0000045557.15923.96

Roemer, L., Lee, J., Salters-Pedneault, K., Erisman, S., Orsillo, S., \& Mennin, D. (2009). Mindfulness and Emotion Regulation Difficulties in Generalized Anxiety Disorder: Preliminary Evidence for Independent and Overlapping Contributions. Behavior Therapy, 40(2), 142-154. http://dx.doi.org/10.1016/j.beth.2008.04.001 
Shapiro, S., Carlson, L., Astin, J., \& Freedman, B. (2006). Mechanisms of mindfulness. Journal Of Clinical Psychology, 62(3), 373-386. http://dx.doi.org/10.1002/jclp.20237

Stratton, S. P. (2015). Mindfulness and contemplation: Secular and religious traditions in Western context. Counseling and Values, 60(1), 100-118.

Strauss C, Cavanagh K, Oliver A, Pettman D. (2014). Mindfulness-based interventions for people diagnosed with a current episode of an anxiety or depressive disorder: a meta-analysis of randomised controlled trials. PLoS One. 9(4):e96110

Taren, A. A., Gianaros, P. J., Greco, C. M., Lindsay, E. K., Fairgrieve, A., Brown, K. W., ... \& Creswell, J. D. (2017). Mindfulness meditation training and executive control network resting state functional connectivity: A randomized controlled trial. Psychosomatic Medicine, 79(6), 674-683.

Teasdale, J., Segal, Z., Williams, J., Ridgeway, V., Soulsby, J., \& Lau, M. (2000). Prevention of relapse/recurrence in major depression by mindfulness-based cognitive therapy. Journal of Consulting And Clinical Psychology, 68(4), 615-623.

Tennant, R., Hiller, L., Fishwick, R., Platt, S., Joseph, S., Weich, S., ... \& Stewart-Brown, S. (2007). The WarwickEdinburgh mental well-being scale (WEMWBS): development and UK validation. Health and Quality of life Outcomes, 5(1), 63.

Treynor, W., Gonzalez, R., \& Nolen-Hoeksema, S. (2003). Rumination reconsidered: A psychometric analysis. Cognitive therapy and research, 27(3), 247-259.

Vettese, L. C., Toneatto, T., Stea, J. N., Nguyen, L., \& Wang, J. J. (2009). Do mindfulness meditation participants do their homework? And does it make a difference? A review of the empirical evidence. Journal of Cognitive Psychotherapy, 23(3), 198-225. 
Vøllestad, J., Nielsen, M., \& Nielsen, G. (2011). Mindfulness- and acceptance-based interventions for anxiety disorders: A systematic review and meta-analysis. British Journal Of Clinical Psychology, 51(3), 239-260. http://dx.doi.org/10.1111/j.2044-8260.2011.02024.x

Webb, T. L., Miles, E., \& Sheeran, P. (2012). Dealing with feeling: a meta-analysis of the effectiveness of strategies derived from the process model of emotion regulation. Psychological bulletin, 138(4), 775.

Williams M. J., Dalgleish T., Karl A., Kuyken W. (2014). Examining the Factor Structures of the five facet mindfulness questionnaire and the self-compassion scale. Psychol. Asses. 26, 407-418. 10.1037/a0035566

Wilson BM, Mickes L, Stolarz-Fantino S, Evrard M, Fantino E. 2015. Increased FalseMemory Susceptibility After Mindfulness Meditation. Psychol. Sci. 26(10):1567- 73

Zerubavel, N., \& Messman-Moore, T. L. (2015). Staying present: incorporating mindfulness into therapy for dissociation. Mindfulness, 6(2), 303-314. 